\title{
St John's wort is at least as effective as paroxetine in reducing severity of depression and is better tolerated
}

Szegedi A, Kohnen R, Dienel A, et al. Acute treatment of moderate to severe depression with hypericum extract WS 5570 (St John's wort): randomised controlled double blind non-inferiority trial versus paroxetine. BMJ 2005;330:503.

\section{Is St John's wort as effective as paroxetine in people with moderate to severe depression?}

\section{METHODS}

Design: Randomised controlled trial.

Allocation: Concealed.

Blinding: Double blind.

Follow up period: Six weeks.

Setting: Twenty one psychiatric primary care practices in Germany; recruitment May 2000 to July 2003.

Patients: 251 people, aged 18-70 years, with single or recurrent moderate or severe episodes of unipolar major depression, without psychotic features, persisting for two weeks to one year.

D Intervention: $300 \mathrm{mg}$ St John's wort (hypericum extract) three times daily plus placebo once daily; or $20 \mathrm{mg}$ paroxetine once daily plus placebo three times daily. If total depression score had not decreased by at least $20 \%$ after two weeks of treatment, the dose was doubled.

国倩

Outcomes: Symptoms of depression (Hamilton total depression score); adverse events.

al Patient follow up: $82 \%$ completed the acute treatment phase.

\section{MAIN RESULTS}

St John's wort was at least as effective as paroxetine in reducing severity of depression after six weeks (change from baseline in

For correspondence: $M$ Kieser, Dr Willmar Schwabe Pharmaceuticals, PO Box 410925, 76209 Karlsruhe, Germany; meinhard.kieser@schwabe.de

Source of funding: Dr Willmar Schwabe Pharmaceuticals, manufacturer of St John's wort (WS 5570).
Hamilton total depression score: intention to treat -14.4 with St John's wort $v-11.4$ with paroxetine; difference $3.0,97.5 \%$ lower confidence limit 1.5; per protocol -14.6 with St John's wort $v-12.0$ with paroxetine; difference 2.6, 97.5\% lower confidence limit 0.7). Adverse events were less frequent with St John's wort than with paroxetine (69/125 (55\%) with St John's wort $v$ 96/126 (76\%)).

\section{CONCLUSIONS}

St John's wort is at least as effective as paroxetine in reducing severity of depression, and it is better tolerated.

\section{NOTES}

Analyses for the primary outcomes were by intention to treat, however the researchers used one-sided statistical testing to assess non-inferiority of St John's wort.

\section{Commentary}

This randomised controlled trial is one in a long list of studies suggesting that St John's wort extracts are efficacious for depression. Most experts today agree that it is superior to placebo in treating mild to moderate depression. But is it also useful for severe depression and how does it compare with conventional antidepressants? The results of previous trials addressing these questions were not uniform. The current study goes someway in answering them. It strongly suggests that St John's wort also works for people with severe depression. It furthermore shows that it is at least as good as paroxetine, perhaps even better. As the study is of good quality, the results seem reliable.

From a patient point of view, an important reason for opting for the herbal antidepressant is its safety. The current trial and other data show that its adverse effects are similar to placebo. But there is one important caveat: this applies only if it is not combined with other drugs. St John's wort powerfully interacts with a wide range of prescription medicines.

The bottom line is that St John's wort is an efficacious and safe antidepressant-but only if herb drug interactions can be avoided. Therefore, I think, it should not be an over-the-counter medicine as it is presently in the UK, but a drug that should only be taken under close medical supervision.

Professor Edzard Ernst, MD, PhD, FRCP, FRCPEd Director of Complementary Medicine, Peninsula Medical School, Universities of Exeter \& Plymouth, UK 